\title{
Lateral Drift Behavior Analysis in Mixed Bicycle Traffic: A Cellular Automaton Model Approach
}

\author{
Xue Feng, Xi-fu Wang, and Dong-fan Xie \\ School of Traffic and Transportation, Beijing Jiaotong University, Beijing 100044, China \\ Correspondence should be addressed to Xue Feng; fengxue229@126.com
}

Received 12 November 2015; Accepted 5 April 2016

Academic Editor: Anna Vila

Copyright (c) 2016 Xue Feng et al. This is an open access article distributed under the Creative Commons Attribution License, which permits unrestricted use, distribution, and reproduction in any medium, provided the original work is properly cited.

\begin{abstract}
Bicycle movements are always associated with lateral drifts. However, the impacts of lateral drift behavior, as well as variable lateral clearance maintaining behavior due to the variation of drift intensity, on mixed bicycle flow are not clear. This paper establishes a new cellular automata model to study typical characteristics of mixed bicycle traffic induced by lateral drift and its accompanying behavior. Based on derived positive correlation between passing speed and drift speed through survey, the occurrence probability of lateral drift and the degree of maintained lateral clearance are both introduced in accordance with the variance of passing speed. Then, in whole density region, firm conformity between simulation results and actual survey data is reached, which has seldom been achieved in previous studies. It is further verified that speed distortions in intermediate and high density region induced by assumption of constant lateral clearance requirements can be revised by introducing its variability characteristics. In addition, two contrastive impacts of lateral drift behavior are observed. That is, it causes speed fluctuation in low density while alleviating the speed fluctuation in relatively high density. These results are expected to be helpful to improve the simulation performance of mixed bicycle flow as well as depict more realistic vehicle-bicycle conflicts and so on.
\end{abstract}

\section{Introduction}

In urban areas of China, conventional bicycles (abbreviation c-bicycles) as well as electronic bicycles (abbreviation ebicycles) are still prevalent for most short-distance trips in recent years. According to the statistics, these two types of bicycles account for a significant proportion in all travel modes (which varies from $20 \%$ to $50 \%$ [1]). Obviously, an in-depth understanding of bicycle behavior characteristics is the basis of the bicycle traffic management and control. In addition, as major travel modes, which share the same road space with motorized traffic in some specific traffic contexts, an analysis of the mixed bicycle flow dynamics is helpful for scrutinizing the realistic interactions between motorized vehicles and bicycles. Therefore, it is essential to research the characteristics of mixed bicycle flow, and modeling and simulation of mixed bicycle traffic are imperatively important issues for the planning and management of urban traffic systems.

In recent years, as one of the most important microscopic models, cellular automata (CA) model has been widely used in elucidating traffic flows for its conceptual simplicity and fast performance. As for the modeling and simulation of bicycle traffic, two types of frameworks, including the multilane CA and the multivalue CA, have been developed up to now. The first modeling framework works like a CA model of multilane motorized traffic in which a bicycle path also has perceived lanes of travel; that is, the lane-changing rules are used to model lateral movements and the followthe-leader rules are used to describe longitudinal movements, respectively. Then, with the application of this framework, some essential features of both pure and mixed bicycle flows have been captured. For instance, Yang et al. [2] developed a microscopic model based on multilane CA and dynamic floor field to simulate pure bicycle traffic, and more realistic traffic flux-density relations are derived. Simulations of a multilane CA proposed by Jiang et al. [3] demonstrated that some characteristics of the mixed bicycle flow, such as honk effect and segregation effect, are quite similar to those in motorized traffic. Zhao et al. [4] developed a calibrated multilane CA model to predict the number of passing events, and a positive effect of the existence of e-bicycles on increasing passing 
events was verified. Here, a passing event refers to the act by one bicycle to overtake another in front. The number of passing events is always recommended to be used to evaluate the level of service of bicycle facilities. To date, multilane CA model has been applied in a variety of studies to the simulation of bicycle traffic. However, during the modeling procedure, there is usually a lack of attention to a key difference between motorized vehicles and bicycles. In real traffic, motorized vehicle movements are more lane-based; that is, maintaining the desired speed is regarded as the only lanechanging motivation. However, bicycles usually move in an erratic lateral drift manner. Namely, lateral movements also can be performed if their front-gaps are sufficient enough.

As for multivalue CA framework first introduced by Nishinari and Takahashi [5], each cell allows occupation by more than one bicycle and an ultradiscretization of the Burgers equation is applied as the basic evolution equation. Then, several improved versions considering randomization effect or extended maximum speed have been applied to the study of pure or mixed bicycle flow $[6,7]$. Later, for some mixed traffic scenarios with bicycles, such as two-lane road sections with bus stops [8] and vehicle-bicycle shared roads [9], researchers even combined the multivalue CA and the $\mathrm{NaSch}$ model to investigate the influence of bicycle intrusion on motorized traffic. Although multivalue CA model has been demonstrated to suit depicting bicycles' non-lane-based behavior without considering lateral movement rules [6], one crucial defect is the fact that time-space behaviors are hard to be distinguished. Moreover, the length-fixed cell system which cannot be further refined brings difficulties in enhancing the resolution and revealing diversities of some variables, such as maximum speed and acceleration. Then some microscopic traffic features are more difficult to be scrutinized. These defects also stand as crucial barriers for modeling vehicle-bicycle conflicts. From the above, we can see that the current CA models have some limitations more or less. A proper CA model that can precisely depict bicycles' lateral drift manner and ensure realism of the simulation in different traffic conditions thus needs to be put forward.

We next discuss the lateral clearance maintaining phenomena in mixed bicycle flow. Actually, for both motorized vehicles and bicycles, the requirement of lateral clearance maintained with others always serves as an efficient means for situation awareness and hazard avoidance on roads. As for motorized vehicle traffic, the lateral gap maintaining phenomenon has attracted the attention of many researchers, and several pieces of research have been reported. Singh [10] studied the relationship between the lateral gap and speed of the interacting vehicles based on the field data collected. The results illustrate that larger lateral gaps are required when motorized vehicles are in fast speed state compared with stationary situation. Gunay [11] pointed out that more friction clearances are required as the passing vehicle's speed increases. Furthermore, in mixed traffic contexts, many researchers $[12,13]$ have studied the evolvement of motorized vehicle's speed owing to the influence of bicycles, and positive correlations between the motorized vehicle's passing speed and the lateral safety distance are obtained based on the findings of field studies. With regard to bicycle traffic, a common perspective of existing literatures is that no bicyclist can ride a bicycle in a perfectly straight line, and some clearance is required to avoid lateral collision. To date, due to much smaller size than motorized vehicles, a bicycle's occupancy width is always set as a constant value slightly larger than its actual width in existing models. Nevertheless, the clearance requirement of bicycles is also not invariable as it can be confirmed from many phenomena in reality, such as a bicycle's frequent tendency to move closer to a slower one than a faster one and the constant adaptation of a bicycle's speed affected by the effective route width ahead of it. So, a systematic study of the characteristics and effects of variable lateral clearance maintaining behavior will be a meaningful attempt to elucidate mixed bicycle flow.

A review of relevant research shows that, so far, the impacts of lateral drifts are seldom explored; besides, related research into the variability of lateral clearance maintaining behavior is also lacking. Thus, these limitations motivate us to develop a new CA model incorporating the lateral drift behavior and its consequent variable lateral clearance maintaining behavior to investigate the mixed bicycle flow. Based on the enhanced resolution of the finer cell system and the model calibration obtained from the field investigations in Beijing, China, many important characteristics of mixed bicycle traffic are captured and elucidated. The rest of the paper is organized as follows. In Section 2, the survey method of the bicycle's drift behavior and basic features of data collected are described. In Section 3, the modeling approach is explicated, including the model definition and evolution rules. In Section 4, numerical simulations are performed and property analyses are carried out. Finally, conclusions are summarized and recommendations are made in Section 5.

\section{Survey Method of Passing Speed-Drift Speed Characteristics}

Before the presentation of the model, the distribution of drift speed over space through survey needs to be given for description of drift behavior through CA modeling. However, in this paper, the positive correlation between the passing speed of a motorized vehicle and its lateral safety distance noted previously, as well as the clearance requirement of a bicycle due to the presence of later drift behavior, inspires our study on the relationship between the passing speed and the lateral drift speed in mixed bicycle traffic contexts. Then, if the relationship was verified with field data, on one hand, lateral drift phenomena can be reproduced more precisely; on the other hand, variability of lateral clearance maintaining similar to that of motorized vehicles will be verified, and the influence of this characteristic on mixed bicycle flow can be further investigated in detail.

Therefore, to support our assumption, on clear days between September 10 and September 25, 2014, traffic survey was taken on selected road sections of Chegongzhuang Street and Pinganli Street in Beijing, China. Since these road sections contain separated bicycle paths and footbridges, bicycles cannot be affected by motorized vehicles and locations for video cameras are conveniently available. The survey was 


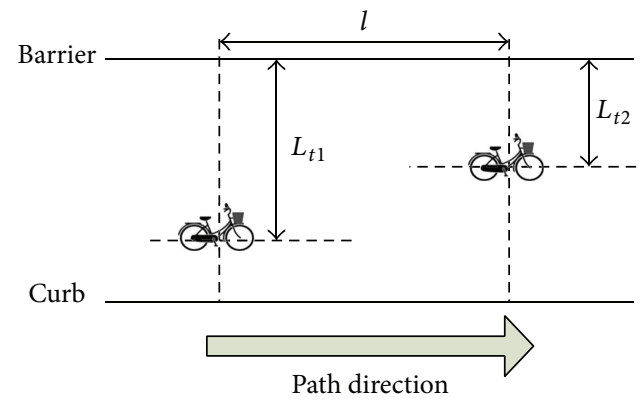

FIGURE 1: Survey methodology of collecting passing speed and drift speed.

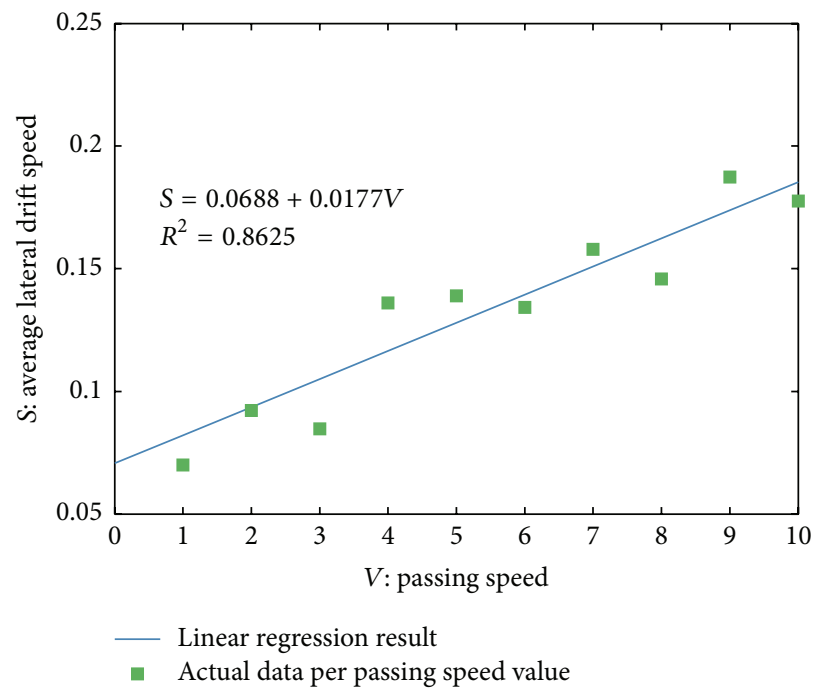

FIGURE 2: Linear regression result.

carried out by the following method. As shown in Figure 1, specifying a constant road section with a length of $l$, the bicycle's passing speed and lateral drift speed are calculated by $v_{p}=l /\left(t_{2}-t_{1}\right), v_{l}=\left(L_{t 2}-L_{t 1}\right) /\left(t_{2}-t_{1}\right)$. Here, $L_{t 1}$ and $L_{t 2}$ denote the lateral distances to the safety barrier at the arrival time $t_{1}$ and departure time $t_{2}$, respectively.

As the bicycle's passing speed and drift speed are usually affected by nearby neighbors, the analysis includes only samples with sufficient distances around from others. After the data collection process, a total of 300 valuable samples for each bicycle type are obtained and taken into the regression analysis. For the simplicity of the modeling, the drift tendency element on either side of a bicycle is deliberately ignored. Finally, the linear regression result shows a positive correlation between the passing speed and the lateral drift speed (cf. Figure 2). That is, as expected, lateral clearance requirements for bicycles exhibit variability characteristics which are similar to those for motorized vehicles $[12,13]$.

\section{Model}

In this section, a new CA model for mixed bicycle flow considering the lateral drift behavior and the accompanying variable clearance maintaining behavior is proposed. Because the appropriate design of cell size is crucial to depict the mixed bicycle flow dynamics, two factors, including the bicycle's physical size and the two-dimensional moving speeds, are both considered for the cell size design. Consequently, to reflect observed maximum lateral drift speed (related to the cell width $W_{c}$ ) and cope with minimum acceleration requirement (related to the cell length $L_{c}$ ), a fine cell of $1 \mathrm{~m} \times$ $0.25 \mathrm{~m}$ is defined as the common unit in this paper. Then the lateral drift behavior is represented by displacing one cell laterally according to the occurrence probability of random choice for drift $p_{\mathrm{drift}, t}$ in each time step. Here, $p_{\mathrm{drift}, t}$ is set as $\left(\alpha+\beta v_{i, t}\right) / W_{c}$ based on the regression result, and $\alpha$ and $\beta$ are the scaling parameters as shown in Figure 3. In addition, a new occupancy rule considering variable lateral clearance is introduced in this paper. According to the actual length of a typical bicycle, the bicycle length is set as 2 cells. The occupied width $W_{b}$ consists of physical width $w$ and variable lateral clearances $c_{i, t}$ on the two sides of the bicycle.

Then, similar to other variables in CA model, including space, speed, and time, lateral clearance should be treated as a discrete variable. The weighting variable $\mu$ is introduced to measure the bicycle's sensitivity degree of lateral drift and 


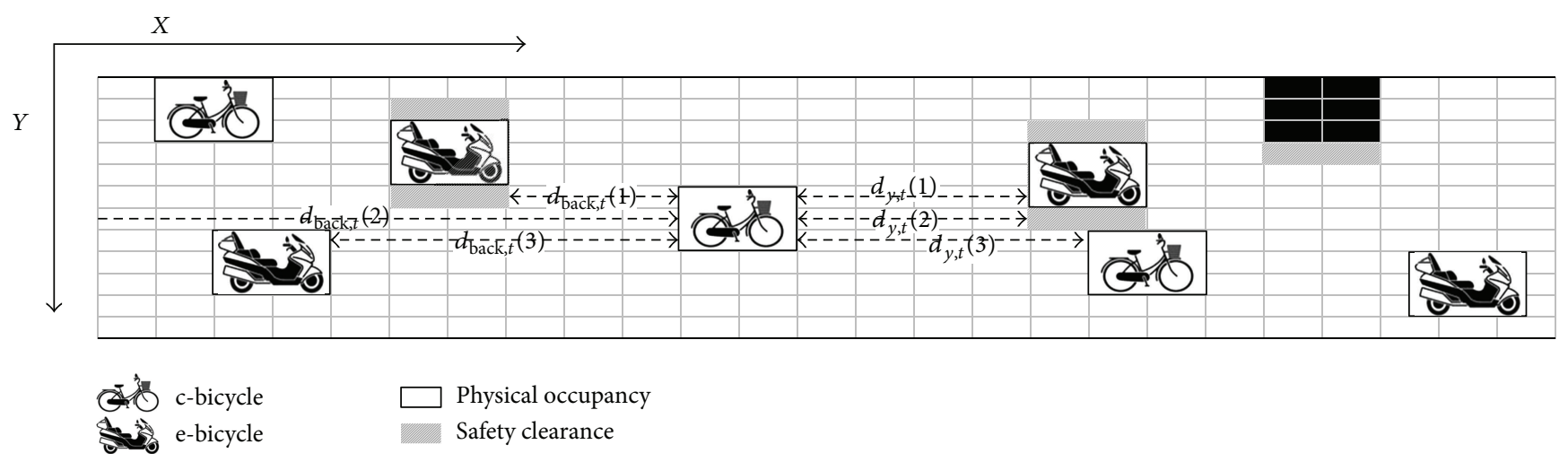

FIgURE 3: Minimum front-gap and back-gap evaluated by a c-bicycle to perform lateral movement.

taken as a constant value smaller than 1 . Then, there are two value options $(0,1)$ available for lateral clearance in this paper. Thus, the lateral clearance $c_{i, t}$ of bicycle $i$ at time $t$ is set as

$$
c_{i, t}= \begin{cases}1, & \text { if } \operatorname{rand}() \leq \frac{\mu p_{\mathrm{drift}, t}}{2} \\ 0, & \text { else. }\end{cases}
$$

Moreover, it is assumed that there is no influence of road markings on the movement of bicycles. Then, in our model, bicycle $i$ can move alongside a road marking without considering its lateral distance from the marking (e.g., the bicycle marked in black in Figure 3).

Owing to two-dimensional movements of bicycles, the update step in this CA model can be defined as two substeps: all the bicycles first update their lateral positions according to lateral movement rules and then decide their forward movements according to forward movement rules.

3.1. Lateral Movement Rules. Let $(x, y)$ be the position of bicycle $i$ at time $t$. $v_{\text {lat }}^{\max }$ denotes the maximum lateral speed of the bicycle, and $v_{i}^{\max }$ and $a_{i}$ denote the maximum passing speed and the acceleration of bicycle $i$, respectively. $d_{y, t}$ represents bicycle $i$ 's front-gap at current position, while in target position $y+k, d_{y+k, t}$ and $d_{\text {back, } t}$ represent its frontgap and back-gap and $d_{\text {safe }}$ represents the safe back-gap. The evaluated procedures of front-gap and back-gap are depicted graphically in Figure 3. $Y_{t+1}^{i}, Y_{t+1}^{h}$, and $Y_{t+1}^{d}$ denote the sets of lateral position available at time $t+1$ when considering incentive criterion, honk effect, and lateral drift behavior, and they are initialized to be empty. $N_{i}, N_{h}$, and $N_{d}$ denote the number of elements in $Y_{t+1}^{i}, Y_{t+1}^{h}$, and $Y_{t+1}^{d}$, respectively. $p_{x}$ represents the probability to choose the position $x$ in next time step, while $p^{i g}$ expresses the probability that the bicycle ignores to move laterally. Then, to be more in line with the realistic bicycle behaviors, three types of lateral movement rules for describing incentive criterion, honk effect, and lateral drift behavior are introduced as follows.

(1) Rules for Describing the Incentive Criterion. Generally, bicyclists tend to ride at expected and constant speeds. Once the front-gap at current position is insufficient to support the desired speed, a bicycle may take lateral movement to look for a longer front-gap. Then, rules for describing this incentive criterion are presented as follows:

(1) If $d_{y, t}<\min \left(v_{i, t}+a_{i}, v_{i}^{\max }\right), d_{y+k, t}>d_{y, t}\left(k \in\left\{-v_{\mathrm{lat}}^{\max }\right.\right.$, $\left.\left.-v_{\text {lat }}^{\max }+1, \ldots,-1,1, v_{\text {lat }}^{\max }-1, v_{\text {lat }}^{\max }\right\}\right), d_{\text {back }, t}>d_{\text {safe }}$, then $y+k \in Y_{t+1}^{\mathrm{i}}$.

(2) After checking all the possible lateral positions, if $Y_{t+1}^{\mathrm{i}}=\varnothing$, then $p_{y}=1$; otherwise, $p_{y}=p^{i g}$ and for each position $y+k^{\prime} \in Y_{t+1}^{i}, p_{y+k^{\prime}}=\left(1-p^{i g}\right) / N_{i}$.

Furthermore, based on the principle of collision-free, if $y+k \notin Y_{t+1}^{i}$, further positions from $y+k$ on the same side are unavailable and should be reasonably eliminated.

(2) Rules for Considering the Honk Effect. Owing to the differences in maximum speed and overtaking requirement between two bicycle types, an e-bicycle will honk the horn with probability $p^{h}$ when there is a hindrance by a preceding c-bicycle and a consequent failure to move laterally. Then, let $h_{i}(t)$ be the honk's state of the e-bicycle $i$ at time $t . h_{i}(t)=1(0)$ means the honk is on (off). Rules for describing the honk motivation can be formulated as follows:

(1) $d_{y, t}<\min \left(v_{i, t}+a_{e}, v_{e}^{\max }\right), Y_{t+1}^{i}=\varnothing, \operatorname{rand}()<p^{h}$, then, $h_{i}(t)=1$; otherwise, $h_{i}(t)=0$.

If stimulated by honks, c-bicycles usually make way for the following e-bicycles due to their different driving characteristics. Thus, one of the lateral positions with sufficient front-gaps and without blocking the way will be chosen randomly as the target position. Let $y+k$ represent a lateral position that ordinates of c-bicycle $j$ 's occupancy are different from those of e-bicycle $i$ 's occupancy. Rules for describing the c-bicycle's reaction to its following e-bicycle's honk are as follows:

$$
\begin{gathered}
\text { (2) } d_{y, t} \geq \min \left(v_{j, t}+a_{c}, v_{c}^{\max }\right), d_{y+k, t} \geq \min \left(v_{j, t}+a_{c},\right. \\
\left.v_{c}^{\max }\right), d_{\text {back }, t}>d_{\text {safe }}, \text { then } y+k \in Y_{t+1}^{h} .
\end{gathered}
$$


(3) If $Y_{t+1}^{h}=\varnothing$, then $p_{y}=1$; otherwise, $p_{y}=p^{i g}$ and for each position $y+k^{\prime} \in Y_{t+1}^{h}, p_{y+k^{\prime}}=\left(1-p^{i g}\right) / N_{h}$.

(3) Rules for Describing the Lateral Drift Behavior. In order to replicate the bicycle's erratic riding manner, displacing one cell laterally, if situations allow, is characterized as a bicycle's lateral drift behavior in our proposed model. Thus, rules for describing the lateral drift behavior are as follows:

(1) If $d_{y, t} \geq \min \left(v_{i, t}+a_{i}, v_{i}^{\max }\right), d_{y+k, t} \geq d_{y, t}(k=-1,1)$, $d_{\text {back, } t}>d_{\text {safe }}$, then $y+k \in Y_{t+1}^{d}$.

(2) If $Y_{t+1}^{d}=\varnothing$, then $p_{y}=1$; otherwise, $p_{y}=N\left(1-p_{\text {drift }}\right)$, and for each position $y+k^{\prime} \in Y_{t+1}^{d}, p_{y+k^{\prime}}=N p_{\text {drift }} / 2$. Here, $N$ is a normalization factor to ensure $\sum_{k} p_{i+k}+$ $p_{i}=1$.

Since all the bicycles are updated simultaneously, a conflict will occur when several bicycles try to move to overlapped cells in next time step. In order to avoid this situation, one of them will be selected randomly to perform its requested lateral displacement.

3.2. Forward Movement Rules. In this paper, the VelocityEffect (VE) model [14] is employed to determine the bicycle's forward movement, as the speed effect of the preceding vehicle is considered and the obtained flow characteristics are effectively consistent with those in the real traffic. However, since one bicycle may have more than one predecessor due to its non-lane-based manner, we improve the VE model and describe the bicycle forward movement as follows:

(1) acceleration: $v_{i, t+1}=\min \left(v_{i, t}+a_{i}, v_{i}^{\max }\right)$;

(2) deceleration: $v_{i, t+1}=\min \left(v_{i, t+1}, d_{i, t}+\min \left(v_{k, t+1}^{\prime}\right)\right)$;

here, $d_{i, t}$ denotes the minimum front-gap between bicycle $i$ and its predecessors at current position at time $t$; bicycle $k$ denotes one of the predecessors of bicycle $i$; let $v_{k, t+1}^{\prime}$ and $v_{k, t+1}^{d}$ be the imaginary and the desired speeds of bicycle $k$ at time $t+1$, given as follows:

$$
\begin{aligned}
& v_{k, t+1}^{\prime}=\max \left(v_{k, t+1}^{d}-a_{k}, 0\right), \\
& v_{k, t+1}^{d}=\min \left(v_{k}^{\max }, v_{k, t}+a_{k}, d_{k, t}\right) ;
\end{aligned}
$$

(3) randomization: $v_{i, t+1}=\max \left(v_{i, t+1}-a_{i}, 0\right)$ with probability $p^{\mathrm{dec}}$

(4) position update: $x_{i, t+1}=x_{i, t}+v_{i, t+1}$.

Here, $p^{\text {dec }}$ denotes the probability of random slowdown.

\section{Simulations and Analyses}

In this paper, in addition to the conventional indexes including the bicycle flux and the passing speed, the number of passing events is employed as another index to evaluate mixed bicycle traffic in this paper. Let $m$ be the total number of bicycles on the bicycle path. The number of passing events $N_{p}$ can be recorded by the following program:

$$
\begin{aligned}
& \text { for } i=1: m \\
& \qquad \begin{array}{r}
\text { if } x_{i, t-1} \leq x_{n, t-1} \text { and } x_{i, t}>x_{n, t} \\
\qquad N_{p}=N_{p}+1 ;
\end{array} \\
& \text { end }
\end{aligned}
$$

end

In this section, a series of numerical simulations are performed under periodic boundary condition. In the simulations, the length and width of the bicycle path are $L=$ 1000 cells and $W=12$ cells, corresponding to $1 \mathrm{~km}$ and $3 \mathrm{~m}$, respectively. According to our field observations, the maximum speeds of c-bicycle and e-bicycle are set as 4 cells/s and 6 cells/s, respectively. Other model parameters are set as follows: $v_{\text {lat }}^{\max }=4$ cells $/ \mathrm{s}, a_{e}=2$ cells $/ \mathrm{s}^{2}, a_{c}=1 \mathrm{cell} / \mathrm{s}^{2}$, $p^{\text {dec }}=0.05, \mu=0.5, p^{i g}=0.05, p^{h}=0.65$, and $d_{\text {safe }}=2$ cells. In addition, considering no-lane-based bicycle movements in practice, the bicycle number per unit path area at a given time is taken as the density of mixed bicycle flow for the following analysis. In the initial state, c-bicycles and e-bicycles are well mixed and randomly distributed on the path. The number of each bicycle type is decided by the density $\rho$ and e-bicycle proportion $R_{e}$, simultaneously. The simulations last for 50000 time steps. The first 30000 time steps are discarded to avoid transient behavior, and the results are obtained by averaging over 20000 time steps.

In order to verify the rationality and suitability of the proposed model, the simulation results and the actual survey data of the speed-density relation under different e-bicycle proportions are plotted in Figure 4. Field data collected in Nanjing and Ningbo of China in the literature [1] (cf. Figure 4 in [1]) are adopted in this paper. We can see that, for each diagram, the simulation results are effectively consistent with the actual survey data through the whole density region. However, results in existing CA models $[1,15]$ only conform to the survey data in certain density scopes. Thus, the validity of our CA model, as well as the advantage of its simulation performance, can be proved by this specific comparison.

4.1. Effect of the Lateral Drift Behavior on the Mixed Bicycle Flow. In this part, in order to analyze the influence of lateral drift on the mixed bicycle flow, the simulation results in the scenario considering the lateral drift behavior (drift scenario) are compared with those in the scenario disregarding the lateral drift behavior (no-drift scenario).

First, traffic flux-density and passing events-density relations with different e-bicycle proportions are plotted and discussed. As can be seen from Figure 5, some characteristics obtained here concur with those in previous CA models [3, 4] in spite of different rules being introduced; for example, the critical density for traffic flux is smaller than that for number of passing events, and the presence of e-bicycles makes a large contribution to passing events, indicating that our model is further validated by previous studies on simulating mixed bicycle traffic.

Besides, many other flow characteristics caused by lateral drift behavior can be observed. When the bicycle density is 


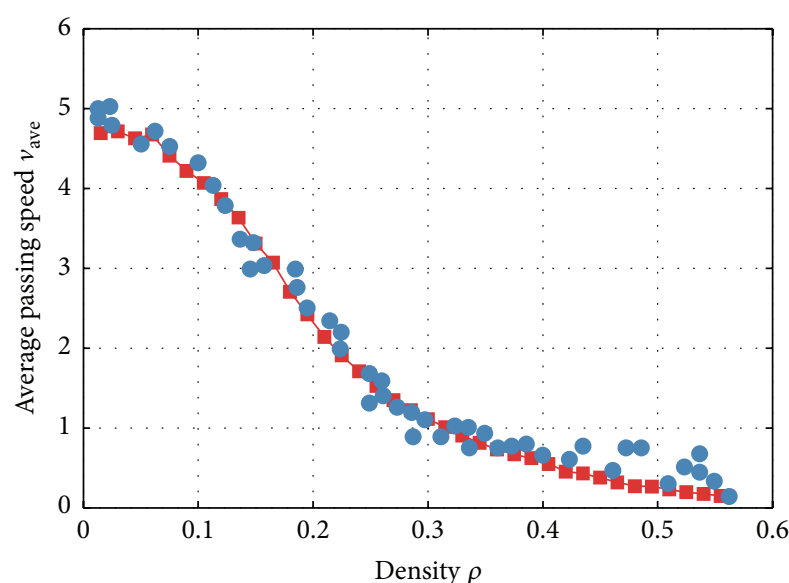

- Simulation results, $R_{e}=0.35$

- Actual survey, $R_{e}=[0.3 \sim 0.4]$

(a)

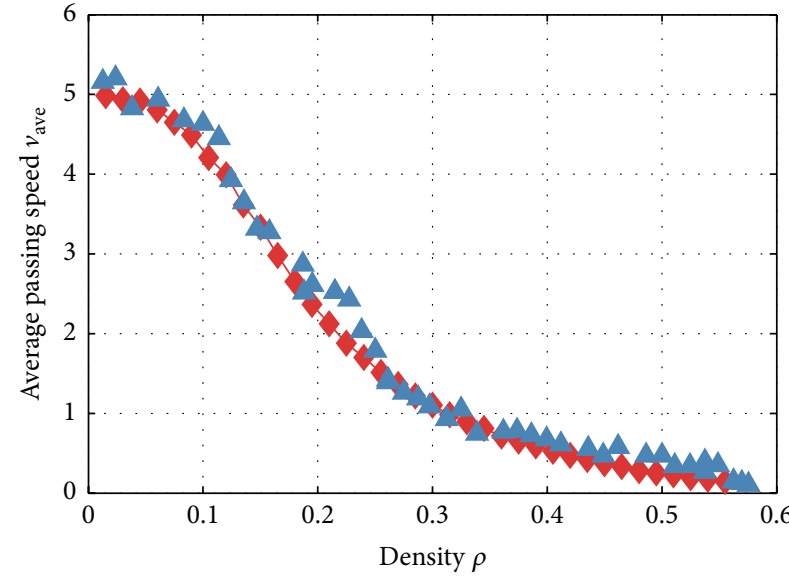

Simulation results, $R_{e}=0.35$

Actual survey, $R_{e}=[0.3 \sim 0.4]$

(b)

Figure 4: Simulation data and survey data of speed-density relations with different e-bicycle proportions.

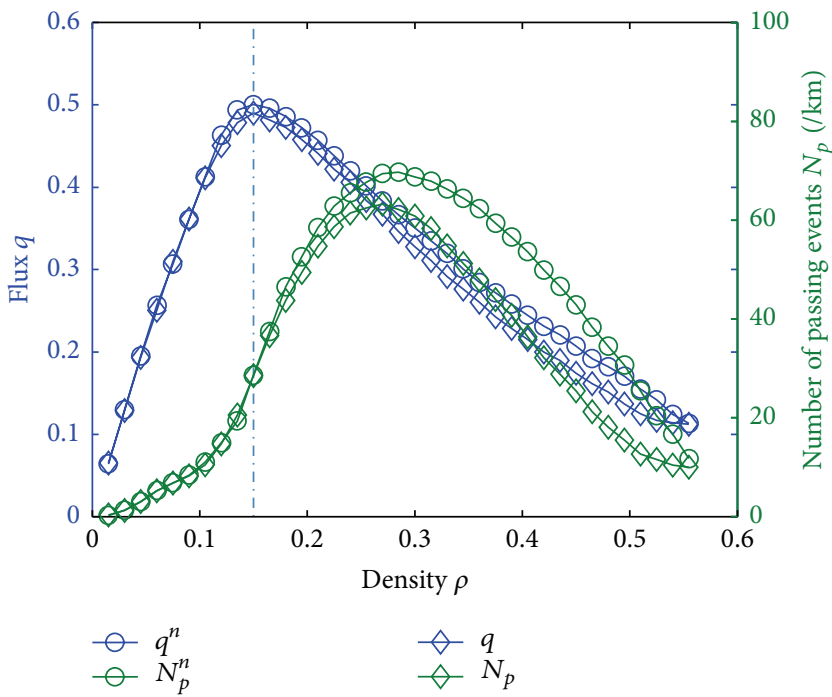

(a) $R_{e}=0.2$

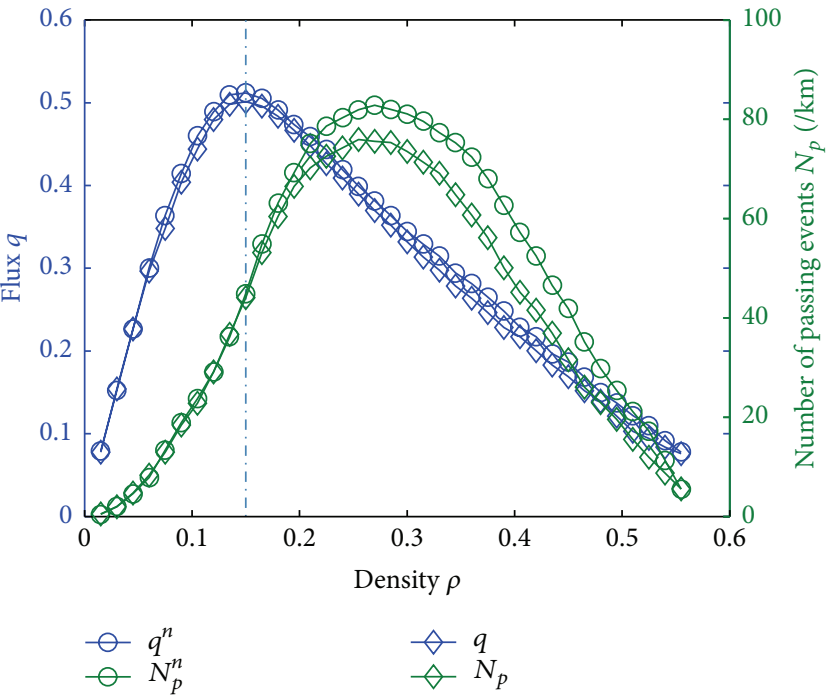

(b) $R_{e}=0.6$

FIGURE 5: The variance of traffic flux $q$ and number of passing events $N_{p}$ with the increase of density $\rho$ (where the superscript $n$ denotes the same parameter in the no-drift scenario).

relatively low, it can be seen that the flux values with and without drifts tend to be the same, while in intermediate and high density region, the flux in drift scenario is generally lower than no-drift scenario at the same density level. Combined with the variance of lateral movement frequency under different densities and scenarios shown in Figure 6, these phenomena can be analyzed as follows: in low density, owing to sufficient moving space provided on the path, bicyclists can move flexibly with frequent lateral movements to avoid being impeded by their predecessors' drifts. Thus, the frequency of lateral movements satisfying incentive criterion (abbreviation incentive lateral movement) with drifts is higher, but no obvious difference of traffic flux between two scenarios is observed. However, as the increase in the bicycle density leads to the decrease in the driving space, incentive lateral movements are more difficult to be performed and more insufficient to compensate for the speed loss induced by lateral drifts' hindrance. With this reason, traffic fluxes in drift scenario are relatively small in intermediate and high density region.

We next focus on the variation of the number of passing events with bicycle density under different e-bicycle proportions. As is shown in Figure 5, it can be seen that the number of passing events with drifts conforms to that without drifts when the bicycle density is low. However, there are two different effects induced by lateral drift behavior worth 


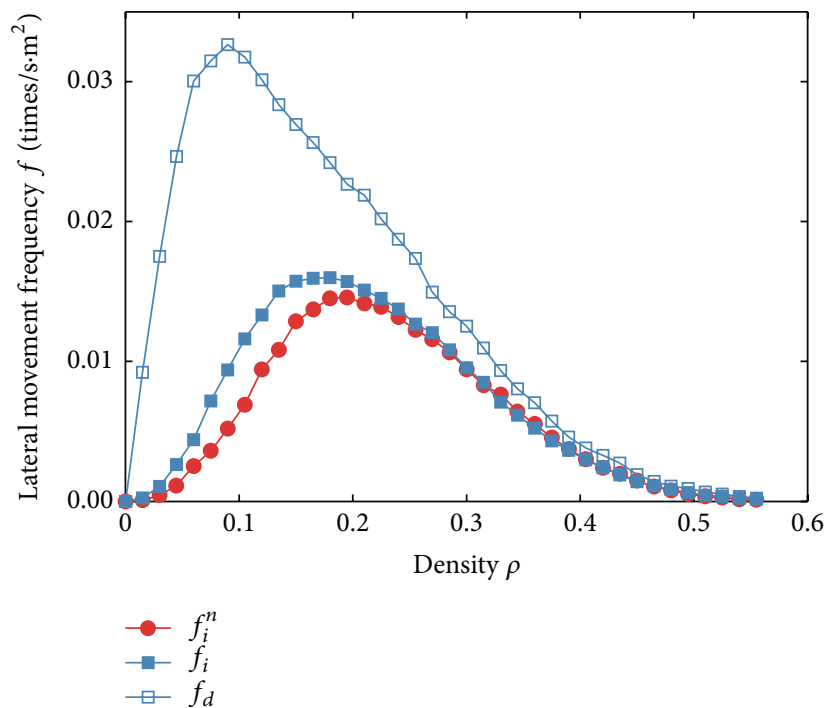

Figure 6: The variance of lateral movement frequency $f$ with the increase of density $\rho$ when $R_{e}=0.2$. (Circle line denotes the frequency of incentive lateral movement $f_{i}^{n}$ in no-drift scenario. Solid and hollow square lines denote the frequencies of incentive lateral movement $f_{i}$ and lateral drift $f_{d}$ in drift scenario, resp.).

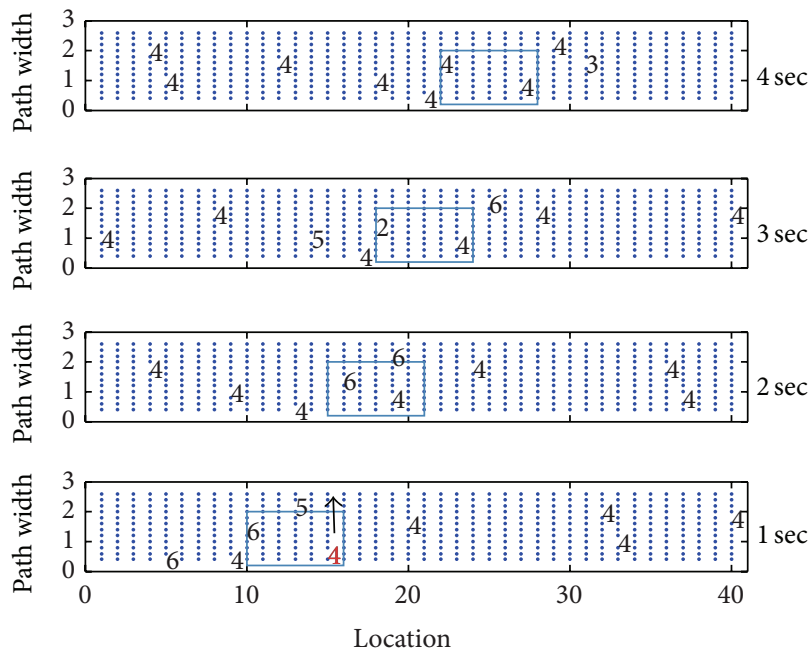

(a)
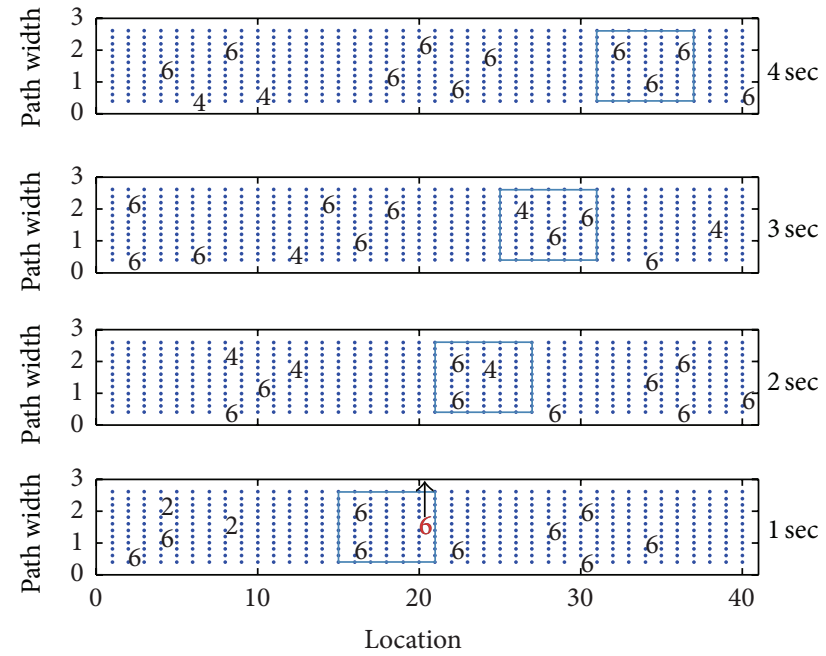

(b)

FIgURE 7: Two different effects induced by lateral drift behavior.

noting in the simulating process. As shown in Figure 7, lateral drift behavior can lead to the undesired low-speed motion that makes the bicycle easily overtaken by followers or delayed to overtake its predecessors within a short period. Thus, although drifts inevitably lead to the increase in the mutual interference, no significant difference in the number of passing events is observed. However, in intermediate and high density region, it can be seen that numbers of passing events in drift scenario are generally lower than no-drift scenario. Owing to the decrease of moving space in lateral with the increase of bicycle density, more bicycles have to decelerate to cope with drifting predecessors, rather than move laterally to overtake. Besides, as one bicycle has larger probability to have more than one follower with the increase of density, bicycles traveling side by side are more likely influenced by the same drifting predecessor and consequently more difficult to overtake each other. For these reasons, in intermediate and high density region, numbers of passing events are lower in drift scenario.

To further investigate the influence of lateral drift behavior on the temporal-spatial dynamics of mixed bicycle flow, time-space diagrams for both with and without drifts under different bicycle densities are shown in Figure 8. Then, some interesting phenomena are observed. In the small density, compared with relatively smooth trajectories without drifts, disorder appears and local clusters can be observed in mixed bicycle flow of drift scenario. However, in high density, the mixed bicycle flow without drifts evolves into the state where 


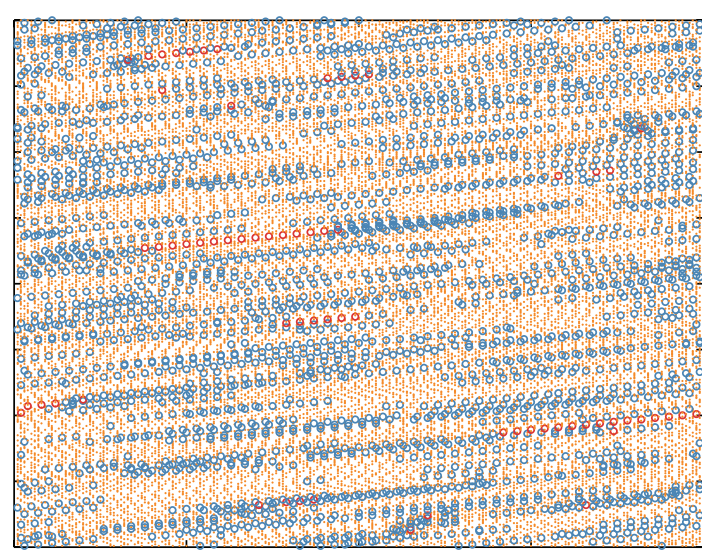

(a)

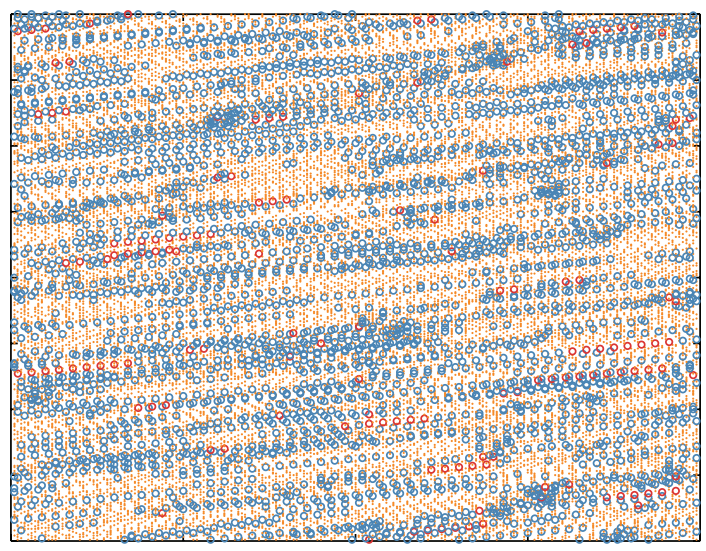

(c)

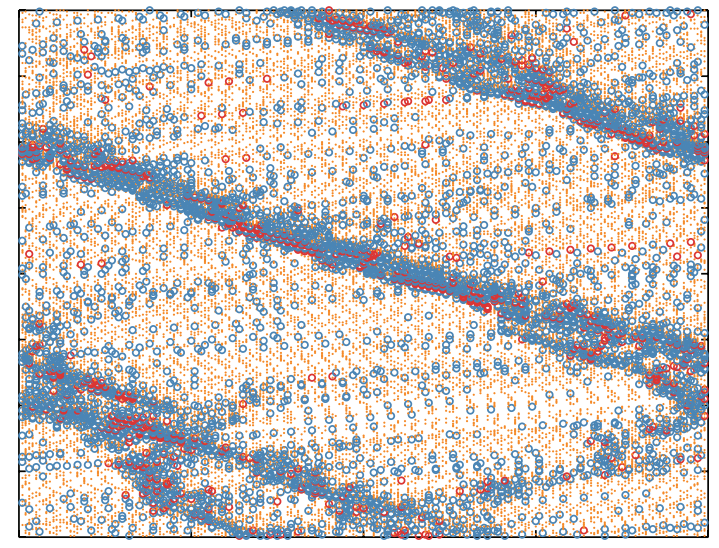

(b)

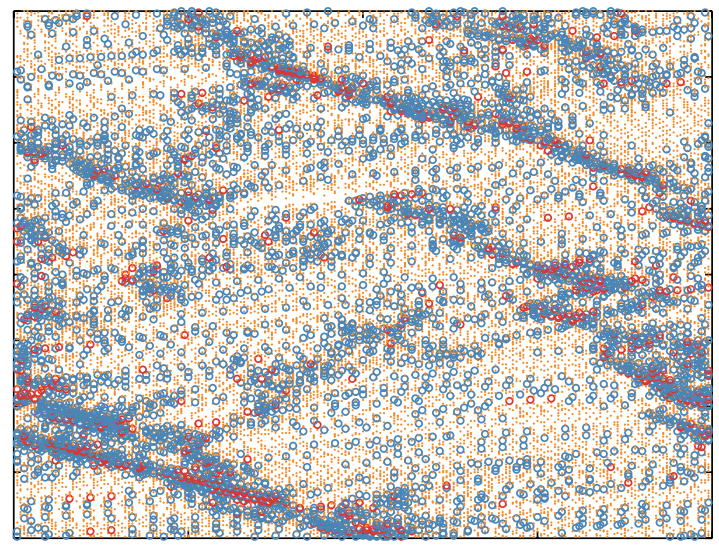

(d)

FIGURE 8: Time-space diagrams of mixed bicycle flow with $R_{e}=0.1$ and (a), (c) $\rho=0.12$ and (b), (d) $\rho=0.16$ in no-drift scenario (a), (b) and in drift scenario (c), (d). (The lateral direction (from left to right) is space and the vertical direction (from bottom to top) is time. Yellow dot, green circle, and red circle denote 1, 2, and 3 bicycles per one-meter-long path resp.).

moving jams coexist with free flow. As for the scenario with drifts, moving jams are lighter and bicycles are distributed in a relatively even state. To further explain these phenomena, passing speed evolvements of the first c-bicycles in simulation scenarios of Figure 8 are plotted in Figure 9. In low density, as occurrence probabilities of hindrances experienced by bicycles are enhanced by drifts, bicycles are more likely forced to slow down. Thus, passing speed oscillates more obviously and trajectories are twists and turns in drift scenario. On the other hand, when the slow-to-start effect is generated in high density, bicycles within start waves decelerate more frequently because of drifts and are delayed to meet moving jams; namely, the speed reduction due to drifts weakens the headway fluctuation. Thus, in drift scenario, moving jams are more difficult to be formed and the distribution of bicycles is more stable and uniform.

\subsection{Effect of the Variable Lateral Clearance Maintaining} Behavior on the Mixed Bicycle Flow. In this part, to analyze the effect of variable lateral clearance maintaining behavior on mixed bicycle flow, a comparison of the speed-density characteristics among simulation results under different lateral occupancy rules is shown in Figure 10. Red plots indicate results with variable lateral occupancy introduced in our paper (variable occupancy rule), while blue and green plots indicate simulation results under different fixed occupancies. The former (no-clearance occupancy rule) ignores lateral clearance and $W_{b}=3$, while the latter (coarse occupancy rule) incorporates a fixed clearance and $W_{b}=4$.

From Figure 10, we can see that when the bicycle density is low $\left(\rho \leq 0.08 \mathrm{bic} / \mathrm{m}^{2}\right)$, both passing speed values, with and without clearance, are basically identical. In this situation, bicycles are in free-driving state and mutual interference is slight; thus speed values are unaffected by the choice of occupancy rule. As density increases, it is observed that the speed on each curve decreases gradually, but the descent range under variable occupancy rule is bigger than that under no-clearance occupancy rule but smaller than that under coarse occupancy rule. These differences can be interpreted as follows: on the one hand, as higher density means higher frequency of touch and mutual interference, bicycles disregarding clearance requirements, that is, moving in incautious manner, are unrealistic, inevitably leading to the speed overestimation in the simulations. On the other hand, due to moving space deficiency, overall passing speed 


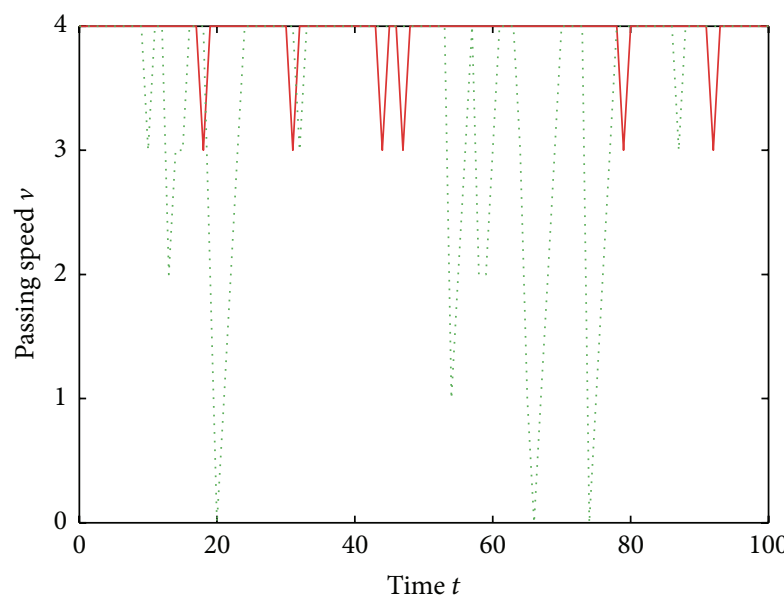

Without drift With drift

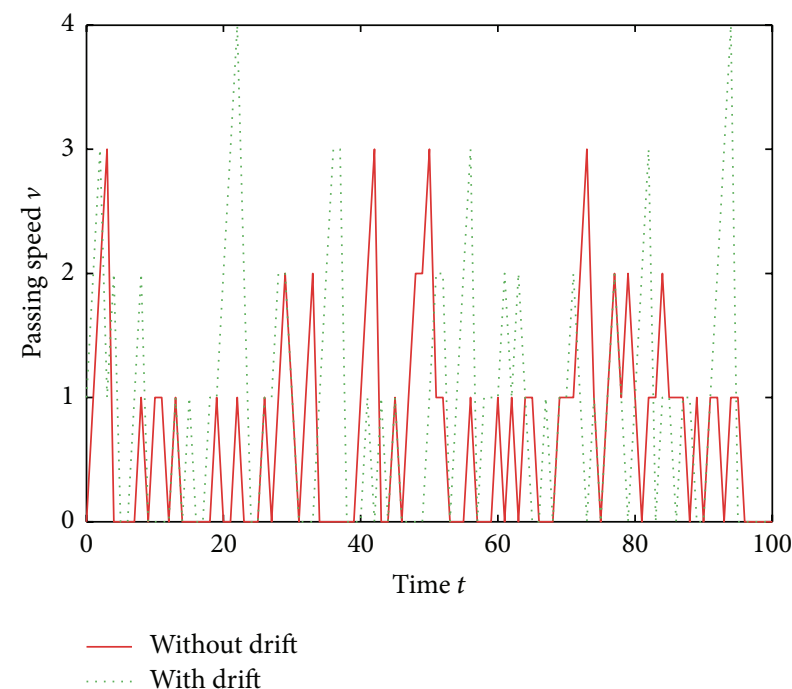

(a) $\rho=0.12$

(b) $\rho=0.16$

With drift

FIGURE 9: Speed distributions of the first c-bicycles in simulation scenarios of Figure 8.

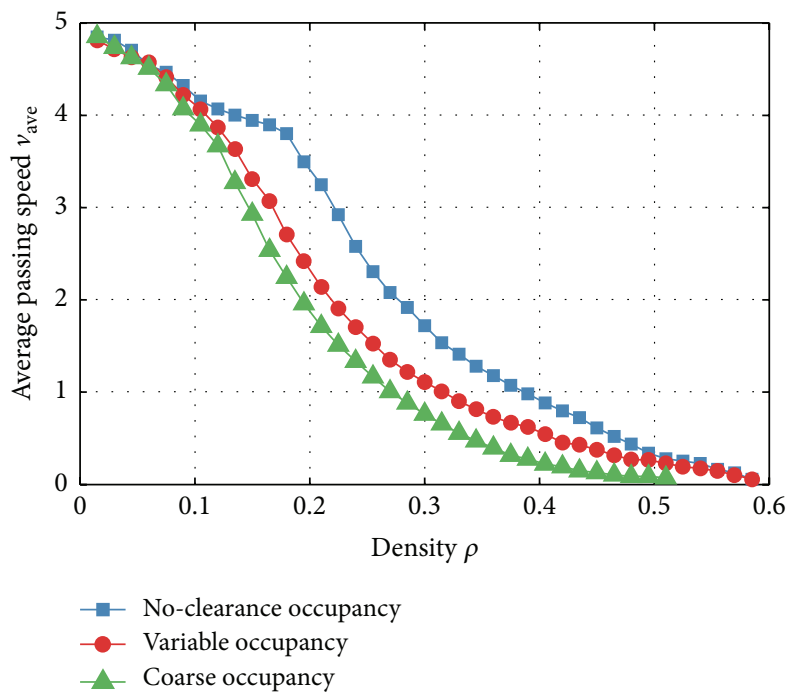

FIGURE 10: Speed-density relations with $R_{e}=0.35$ under different occupancy rules.

decreases significantly as density increases. However, the slower the bicycle is, the lower the pressure to others will be produced and the smaller the lateral clearance will be required; that is, bicyclists in a crowded state can ride much closer than in a free-driving state. This indicates that when coarse occupancy rule is applied, lateral clearances will be more overstated and passing speeds will be predicted more conservatively at higher density. Above comparison thus reveals the importance of introducing variable lateral clearance maintaining characteristic into the proposed model, particularly in intermediate and high density conditions.

\section{Conclusion}

In this paper, the impacts of lateral drift behavior as well as accompanying variable lateral clearance maintaining behavior on mixed bicycle flow dynamics are studied through cellular automata modeling and simulations. To reproduce these two types of behaviors, on the basis of the derived passing speed-drift speed correlation, probabilities of bicycles' random choices for drifting to neighboring positions and maintaining lateral clearances are both introduced and calibrated.

In this research, a series of simulations are performed to investigate characteristics of the mixed bicycle traffic and three types of conclusions are achieved. First, it is verified that the proposed model can accurately describe flow characteristics of the mixed bicycle flow, as a firm conformity between simulated speed values and actual survey data is observed. Second, lateral drift behavior should not be neglected in the modeling process as it creates significant impacts on mixed bicycle traffic, causing speed fluctuation and increasing lateral 
movement frequency in low density region, while reducing traffic flux and passing events and damping speed fluctuation in intermediate and high density region. Third, the introduction of variable lateral distance maintaining behavior will be helpful to revise speed distortions caused by fixed occupancy rules in intermediate and high density region, including the speed overestimation due to the ignorance of lateral clearance and the underestimation of it when considering fixed lateral clearance value. Such findings are expected to capture some valuable characteristics of mixed bicycle traffic and improve the performance of simulation techniques for mixed bicycle flow dynamics as well as vehicle-bicycle conflicts to some extent.

\section{Competing Interests}

The authors declare that there is no conflict of interests regarding the publication of this paper.

\section{Acknowledgments}

This work is partially supported by the National Natural Science Foundation of China (Grants nos. 6127-2029 and 71301007).

\section{References}

[1] S. C. Zhang, G. Ren, and R. F. Yang, "Simulation model of speeddensity characteristics for mixed bicycle flow-comparison between cellular automata model and gas dynamics model," Physica A: Statistical Mechanics and its Applications, vol. 392, no. 20, pp. 5110-5118, 2013.

[2] X.-F. Yang, W. Mao, and Q. Fu, "Modeling of bicycle flow based on dynamic floor field and cellular automata," Acta Physica Sinica, vol. 62, no. 24, Article ID 240511, 2013.

[3] H. Jiang, G. Ren, L. Zheng, J. Chen, and Z. Huang, "Properties analyses for the heterogeneous nonmotorized vehicle traffic based on cellular automaton model," International Journal of Modern Physics B, vol. 28, no. 16, Article ID 1450099, 2014.

[4] D. Zhao, W. Wang, C. Y. Li, Z. B. Li, P. M. Fu, and X. J. $\mathrm{Hu}$, "Modeling of passing events in mixed bicycle traffic with cellular automata," Transportation Research Record, no. 2387, pp. 26-34, 2013.

[5] K. Nishinari and D. Takahashi, "Analytical properties of ultradiscrete Burgers equation and rule-184 cellular automaton," Journal of Physics A: Mathematical and General, vol. 31, no. 24, pp. 5439-5450, 1998.

[6] R. Jiang, B. Jia, and Q.-S. Wu, "Stochastic multi-value cellular automata models for bicycle flow," Journal of Physics A: Mathematical and General, vol. 37, no. 6, pp. 2063-2072, 2004.

[7] B. Jia, X.-G. Li, R. Jiang, and Z.-Y. Gao, "Multi-value cellular automata model for mixed bicycle flow," The European Physical Journal B, vol. 56, no. 3, pp. 247-252, 2007.

[8] X.-M. Zhao, B. Jia, Z.-Y. Gao, and R. Jiang, "Traffic interactions between motorized vehicles and nonmotorized vehicles near a bus stop," Journal of Transportation Engineering, vol. 135, no. 11, pp. 894-906, 2009.

[9] D.-F. Xie, Z.-Y. Gao, and X.-M. Zhao, "Combined cellular automaton model for mixed traffic flow with non-motorized vehicles," International Journal of Modern Physics C, vol. 21, no. 12, pp. 1443-1455, 2010.

[10] B. Singh, Simulation and animation of heterogeneous traffic on urban roads [Ph.D. thesis], Indian Institute of Technology Kanpur, Kanpur, India, 1999.

[11] B. Gunay, "Car following theory with lateral discomfort," Transportation Research Part B: Methodological, vol. 41, no. 7, pp. 722-735, 2007.

[12] Y. J. Luo, B. Jia, J. Liu, W. H. K. Lam, X. G. Li, and Z. Y. Gao, "Modeling the interactions between car and bicycle in heterogeneous traffic," Journal of Advanced Transportation, vol. 49, no. 1, pp. 29-47, 2015.

[13] H.-W. Guo, Z.-Y. Gao, X.-M. Zhao, and D.-F. Xie, "Dynamics of motorized vehicle flow under mixed traffic circumstance," Communications in Theoretical Physics, vol. 55, no. 4, pp. 719724, 2011.

[14] X. B. Li, Q. S. Wu, and R. Jiang, "Cellular automaton model considering the velocity effect of a car on the successive car," Physical Review E, vol. 64, no. 6, Article ID 066128, 2001.

[15] J. Zhang, H. Wang, and P. Li, "Bicycle flow modeling and simulation based on cellular automaton," Journal of Highway and Transportation Research and Development, vol. 23, no. 1, pp. 125-129, 2006. 


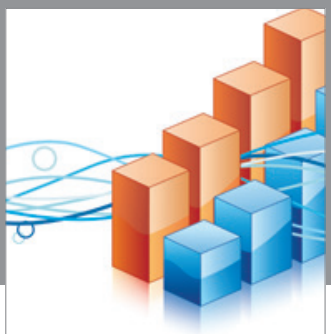

Advances in

Operations Research

vatem alat4

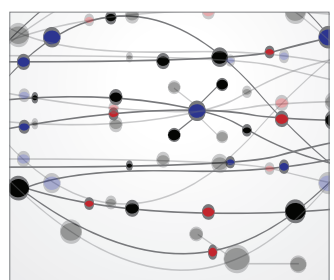

\section{The Scientific} World Journal
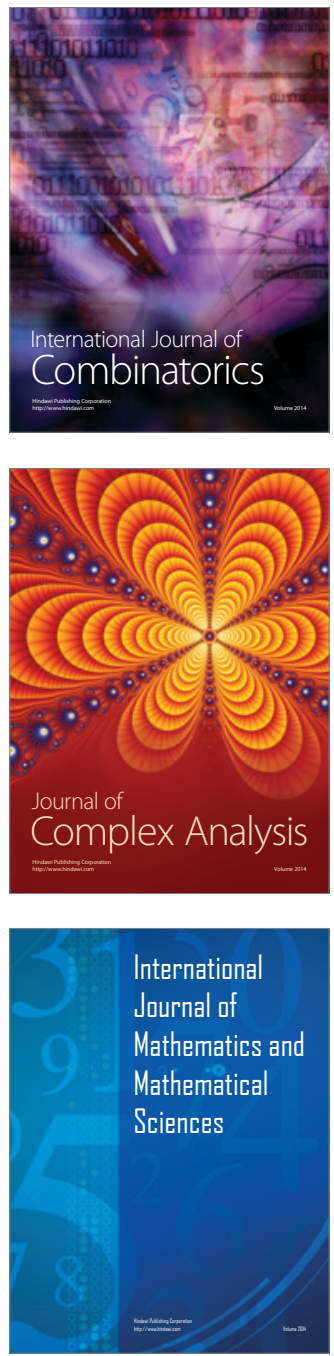
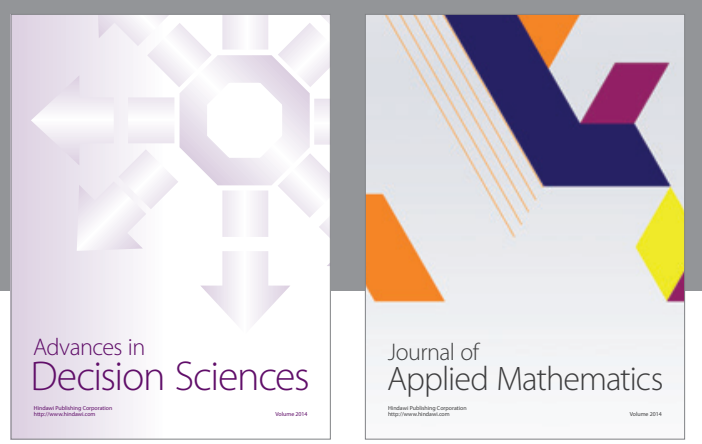

Algebra

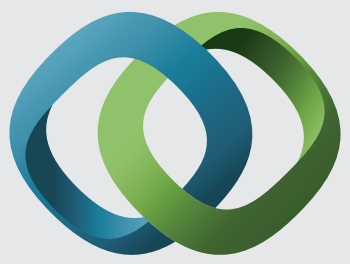

\section{Hindawi}

Submit your manuscripts at

http://www.hindawi.com
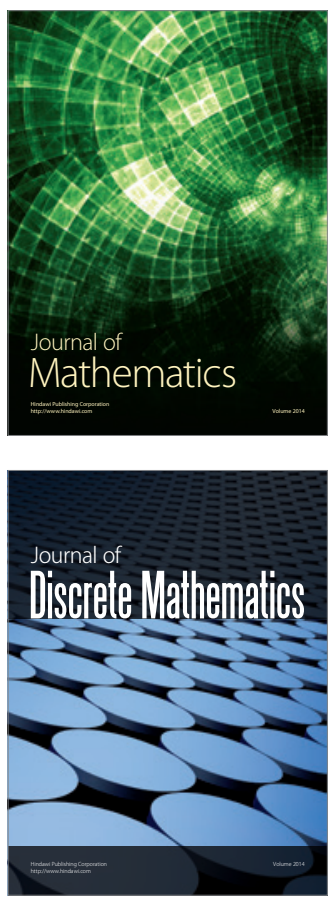

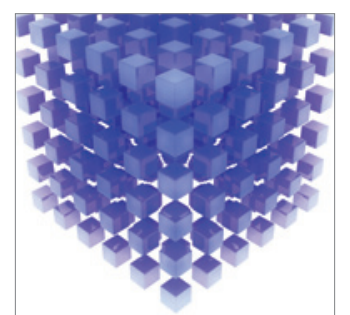

Mathematical Problems in Engineering
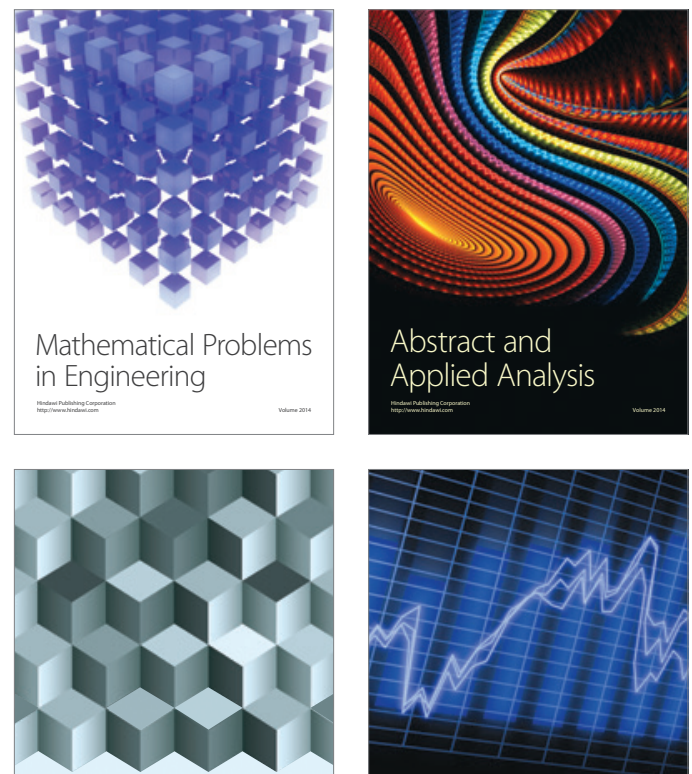

Journal of

Function Spaces

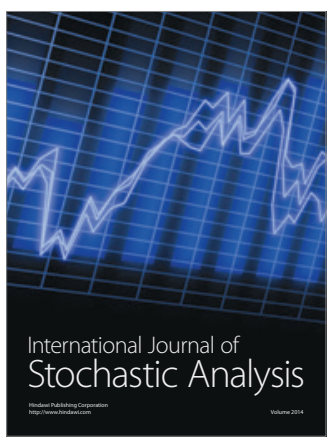

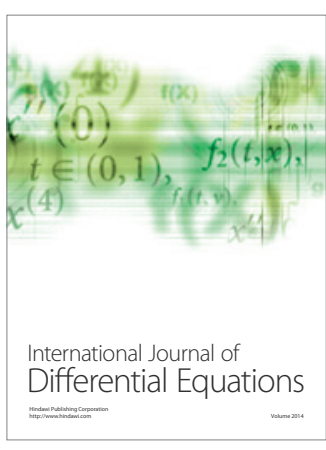
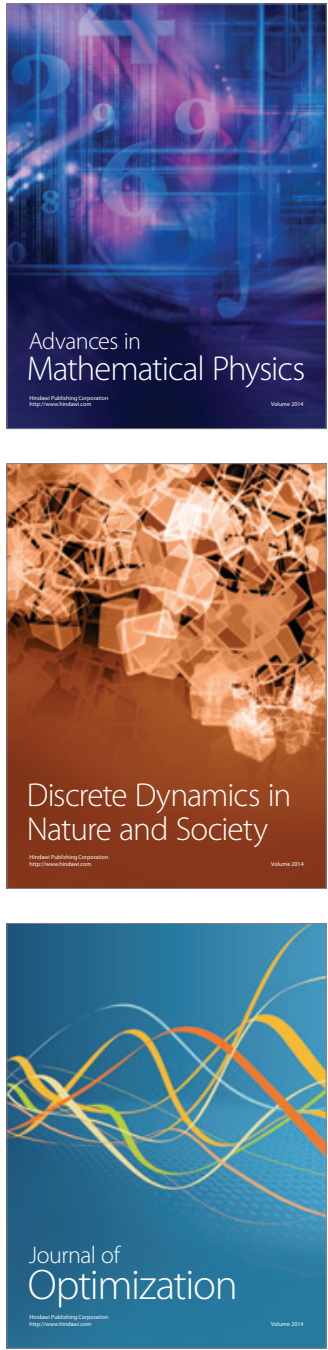